\title{
Maggot Excretions Inhibit Biofilm Formation on Biomaterials
}

\author{
Gwendolyn Cazander MD, Mariëlle C. van de Veerdonk, \\ Christina M. J. E. Vandenbroucke-Grauls MD, PhD, \\ Marco W. J. Schreurs PhD, Gerrolt N. Jukema MD, PhD
}

Received: 28 September 2009/Accepted: 2 March 2010/Published online: 23 March 2010

(C) The Author(s) 2010. This article is published with open access at Springerlink.com

\begin{abstract}
Background Biofilm-associated infections in trauma surgery are difficult to treat with conventional therapies. Therefore, it is important to develop new treatment modalities. Maggots in captured bags, which are permeable for larval excretions/secretions, aid in healing severe, infected wounds, suspect for biofilm formation. Therefore we presumed maggot excretions/secretions would reduce biofilm formation.

Questions/purposes We studied biofilm formation of Staphylococcus aureus, Staphylococcus epidermidis, Klebsiella oxytoca, Enterococcus faecalis, and Enterobacter cloacae on polyethylene, titanium, and stainless steel. We compared the quantities of biofilm formation between the bacterial species on the various biomaterials and the quantity of biofilm formation after various incubation times. Maggot excretions/secretions were added to existing biofilms to examine their effect.
\end{abstract}

Each author certifies that he or she has no commercial associations (eg, consultancies, stock ownership, equity interest, patent/licensing arrangements, etc) that might pose a conflict of interest in connection with the submitted article.

This work was performed at VU University Medical Center.

G. Cazander ( $₫)$, M. C. van de Veerdonk, G. N. Jukema Department of Trauma Surgery, VU University Medical Center, PO Box 7057, 1007 MB Amsterdam, The Netherlands e-mail: G.Cazander@vumc.nl; g.cazander@lumc.nl

C. M. J. E. Vandenbroucke-Grauls

Department of Medical Microbiology \& Infection Control, VU University Medical Center, Amsterdam, The Netherlands

M. W. J. Schreurs

Department of Pathology, VU University Medical Center, Amsterdam, The Netherlands
Methods Comb-like models of the biomaterials, made to fit in a 96-well microtiter plate, were incubated with bacterial suspension. The formed biofilms were stained in crystal violet, which was eluted in ethanol. The optical density (at $595 \mathrm{~nm}$ ) of the eluate was determined to quantify biofilm formation. Maggot excretions/secretions were pipetted in different concentrations to (nonstained) 7-day-old biofilms, incubated 24 hours, and finally measured. Results The strongest biofilms were formed by S. aureus and S. epidermidis on polyethylene and the weakest on titanium. The highest quantity of biofilm formation was reached within 7 days for both bacteria. The presence of excretions/secretions reduced biofilm formation on all biomaterials. A maximum of $92 \%$ of biofilm reduction was measured.

Conclusions Our observations suggest maggot excretions/secretions decrease biofilm formation and could provide a new treatment for biofilm formation on infected biomaterials.

\section{Introduction}

Infections of medical prosthetic devices in trauma and orthopaedic surgery are difficult to treat with conventional therapies like antibiotics and surgery [7], and can lead to severe consequences, such as removal of the implant with functional loss of the affected limb $[18,26]$. The most frequently isolated bacteria causing these implant- and patient-related infections are S. aureus and the coagulasenegative S. epidermidis $[1,11]$. Less common are Pseudomonas aeruginosa, Klebsiella oxytoca, Enterococcus faecalis, and Enterobacter cloacae. In prosthesis-associated infections, the causative bacteria form biofilms composed mainly of a polysaccharide matrix [8]. The most important 
difference between bacteria in biofilms and planktonic microorganisms that can explain the alteration in antibiotic resistance is the metabolic state of biofilm bacteria [24]. The genotype and phenotype of bacteria in biofilms and the protein expression are altered and the oxygen concentration in biofilms is limited [3]. These changes result in metabolic quiescence, which could be overcome by providing alternative electron acceptors of fermentable substrates [3, 24]. Current antimicrobial therapies frequently cannot adequately treat biofilm-associated infections $[8,9,24,26]$ and therefore it is important to develop new treatment approaches to reduce biofilm formation (BF) on biomaterials.

The most important development in the treatment of infectious diseases during the last century was the discovery of penicillin by Fleming in 1928 [29]. At approximately the same time, maggot debridement therapy (MDT) was introduced by William S. Baer, an orthopaedic surgeon who worked at Johns Hopkins Hospital in Baltimore, Maryland [2]. Baer used MDT to treat children with, at that time, incurable osteomyelitis. Although Baer showed the beneficial effects of maggots of the Lucilia sericata, MDT essentially disappeared after the discovery of penicillin, which could control numerous types of severe infections [29]. Because of increasing antibiotic resistance [15], larval therapy was reintroduced in the 1980s as a treatment for severely infected wounds [21]. Maggots now are used successfully in various patient clinics [13, 25]. In 2004, MDT was approved by the US Food and Drug Administration (510[k] \#33391) [27].

In our clinical practice, we use maggots captured in small nylon bags, consisting of a 2-mm thin foam layer of polyvinyl alcohol (Biobag $\AA$; BioMonde $\mathrm{GmbH}$, Barsbüttel, Germany) [13]. These bags are able to permeate maggot excretions and secretions (ES). We have observed healing of wound infections suspect for BF using maggots in such bags. In an earlier pilot study, we found ES reduced the quantity of immature biofilms of P. aeruginosa [6]. However, the current research investigated, for the first time, the influence of a range of concentrations of ES on mature 7-day-old biofilms formed by new, commonly isolated bacterial species on different biomaterials. If maggot ES reduce $\mathrm{BF}$ of bacteria that frequently are isolated from biofilm-associated infections, ES could provide us with a new treatment possibility.

Therefore, we asked (1) whether five bacterial species (S. aureus, S. epidermidis, K. oxytoca, E. faecalis, and E. cloacae), were able to form biofilms on polyethylene (PE), titanium (TI), and/or surgical stainless steel (SSS), and if so, whether (2) the quantity of BF differed among the bacterial species, (3) the quantity of BF depended on the biomaterial surface, and (4) the quantity of biofilms of the biofilm-forming bacteria differed among 3, 5, 7, or
9 days of incubation time. Finally, we hypothesized (5) sterile maggot ES could decrease the growth of the bacterial biofilms on the commonly used biomaterials SSS, TI, and PE.

\section{Materials and Methods}

First, we investigated BF of five different, clinical isolated species on comb-like models of PE, TI, and SSS suspending in a 96-well microtiter plate with nutrient medium and bacteria (Fig. 1) [6]. Two of the five tested bacterial species formed visible biofilms that could be quantified and these bacteria were used to investigate the second to fifth research questions. The dependent variable in the second to fifth research questions was the quantity of formed biofilm; BF was compared among the two biofilm-forming species, among the different biomaterials SSS, TI, and PE and between various incubation times on Days 5, 7, and 9 . Finally, we determined the effect of maggot ES on BF formation.

The custom-made, sterile comb-like models of regularly available orthopaedic implant materials, consisting of eight prongs were produced according to our design. These models of SSS, TI, and PE (Litos GmbH, Hamburg, Germany) were made to fit in a 96-well microtiter plate [6]. Each prong was considered one test and experiments were performed at least 12 times.

S. aureus, S. epidermidis, K. oxytoca, E. faecalis, and E. cloacae all were isolated from infected prosthetic devices of patients in our clinic. BF was tested using a previously described model [6]. Briefly, S. aureus and S. epidermidis were grown overnight in tryptic soy broth (TSB) medium (Becton, Dickinson \& Co, Franklin Lakes, NJ) and K. oxytoca, E. faecalis, and E. cloacae in brain heart infusion (BHI) medium (Becton, Dickinson \& Co) at $37^{\circ} \mathrm{C}$. A stationary phase culture was made in either TSB or BHI to a density of McFarland 0.5 corresponding with $1.5 \times 10^{8}$ colony-forming units (CFUs) per $\mu \mathrm{L}$. The inoculum was controlled by inoculating nutrient agar plates (Biotrading Benelux BV, Mijdrecht, The Netherlands) with $10-\mu \mathrm{L}$ volumes of serial 1:10 dilutions of the bacterial suspension and counting the formed CFUs after incubation overnight at $37^{\circ} \mathrm{C}$.

The bacterial suspension was diluted to a final inoculum of $2.5 \times 10^{5}$ bacteria per $\mathrm{mL}$ and a volume of $100 \mu \mathrm{L}$ of this suspension was pipetted into 24 wells of a sterile 96-well flat-bottomed microtiter plate (Greiner Bio-one $\mathrm{BV}$, Monroe, NC). One comb of each biomaterial was placed in eight of the wells and four of these microtiter plates with combs were incubated for 3, 5, 7, or 9 days at $37^{\circ} \mathrm{C}$ to form biofilms on the devices. Control combs were suspended in nutrient medium. 
Fig. 1 An overview of the study design is shown. $\mathrm{BF}=$ biofilm formation; $\mathrm{PE}=$ polyethylene; $\quad \mathrm{TI}=$ titanium; $\mathrm{SSS}=$ surgical stainless steel; ES = excretions and secretions.

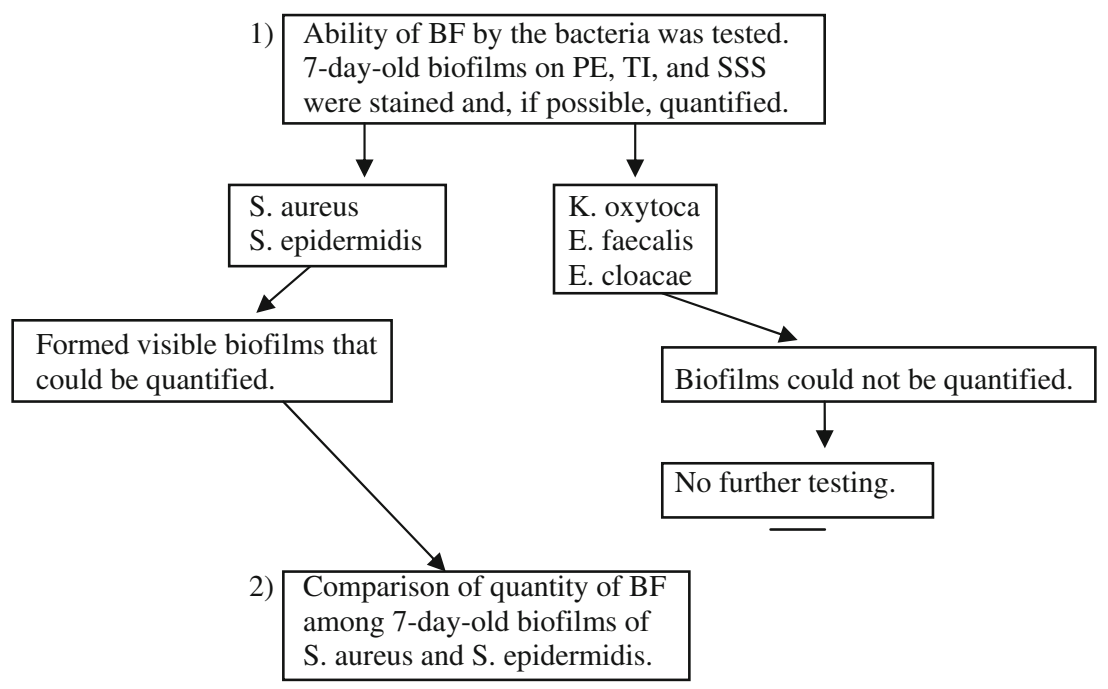

Statistical analysis by Student's T-test for independent variables:

A comparison was made between the mean of the 7-day-old biofilm formed by $S$. aureus and the mean of the 7-day-old biofilm by $S$. epidermidis.

3) Comparison of quantity of 7-day-old biofilms among PE, TI, and SSS.

Statistical analysis by one-way ANOVA, followed by LSD post hoc test:

A comparison was made between the mean of the quantities of 7-day-old biofilms formed on PE, TI, and SSS.

4) Comparison of quantity of biofilm formed after 3, 5, 7, and 9 days.

Statistical analysis by repeated measures ANOVA, followed by Bonferroni correction:

A comparison was made between the mean of the quantities of biofilm at each time.

5) Comparison of quantity of 7-day-old biofilms with and without presence of (different concentrations of) maggot ES.

Inhibition of further biofilm growth was tested (compared with 8-day-old $\mathrm{BF}$ ) and breakdown of existing biofilms (compared with 7-day-old BF).

Statistical analysis by Student's T test for independent variables:

A comparison was made between the mean BF with ES (per concentration) and the mean of the 7-day-old BF and 8day-old BF without ES.

Experiments for research questions 1 to 4 were performed in octuplicate.

Experiments for research question 5 were performed in quadruplicate.

After the incubation time, each comb was washed under slow-running distilled water at room temperature for 30 seconds to remove planktonic cells. All comb models were stained for 15 minutes with $1 \%$ crystal violet [6], based on the methods of Pitts et al. [19] and Stepanovic et al. [23].
Each comb was suspended in eight wells of a new microtiter plate filled with $270 \mu \mathrm{L}$ ethanol absolute to absorb the crystal violet [19]. The plates were left for 48 hours at room temperature to allow the stained biofilm bacteria to elute with ethanol. All stained biofilm bacteria are soluble 
in ethanol, however the biofilm on PE could not be eluted entirely in ethanol (maximum time tested was 96 hours), but we standardized the time of elution to 48 hours to guarantee reliable interpretation of the results. After gentle shaking of the microtiter plate, a volume of $150 \mu \mathrm{L}$ of the ethanol and crystal violet solution was pipetted into a new plate and the optical density (OD) of this solution was measured at a wavelength of $595 \mathrm{~nm}$ and is representative for the quantity of biofilm formation [19]. Biofilms of each bacterial species were formed at least in octuplicate, so that one comb was tested with each of the eight prongs suspended in a separate well with nutrient medium and bacterial suspension.

Sterile maggot ES of Instar-3 larvae (BioMonde $\mathrm{GmbH}$ ) were collected as described previously [6]. Briefly, the maggots were incubated in sterile tubes for 1 hour at $35^{\circ} \mathrm{C}$ in darkness. Then, ES were removed by pipette, divided in aliquots, and stored at $-80^{\circ} \mathrm{C}$. One ES pool was collected from at least 1000 maggots, which resulted in approximately $1200 \mu \mathrm{L}$ ES per hour. The protein concentration was determined using the Pierce Bicinchoninic Acid Protein Assay kit (Pierce Biotechnology, Rockford, IL).

Absolute OD values of the control BF after 7 days without ES are reported in Results. To compute absolute and relative OD, we first subtracted the material-specific background absorbance. The measured OD values of the biofilm experiments were converted to percentages using the following formula:

[OD (biofilm) $\times 100 \%] /$ OD (control)

where OD (biofilm) refers to either BF measured on Day 3, 5 or 9 (without ES) or BF measured after the addition of increasing concentrations of ES and where OD (control) is the BF after 7 days on the negative control prongs. In all experiments, the 7-day-old biofilm without ES (the control) was considered $100 \%$.

Biofilms were allowed to form on 13 combs of each of the materials for 7 days, two other combs were suspended in nutrient medium without bacteria as negative controls and represented the material-specific background absorbance. BF on two of the 13 combs was measured on Day 7. Two other combs were suspended in a new microtiter plate with medium and incubated another 24 hours to allow additional growth of the biofilm (the 8-day-old biofilms); nine combs were suspended in a logarithmic range of concentrations of ES, diluted in nutrient medium: 0.31, $0.93,2.78,8.33,25$, and $75 \mu \mathrm{g}$ ES per well. All wells were incubated another 24 hours at $37^{\circ} \mathrm{C}$ and then we quantified the biofilm as described previously. This experiment investigated possible biofilm inhibition and breakdown by ES. Biofilm inhibition (or prevention) by ES was defined as $\mathrm{BF}$ that was lower than 8-day-old biofilms which grew further after Day 7. BF that was lower than 7-day-old BF after incubation in ES was defined as biofilm breakdown. Biofilm reduction is any effect of ES that results in less BF.

The first research question was not analyzed statistically because the results were obtained by observation only. For the second and the last research questions, Student's t test for independent groups was used. The means of the 7-dayold biofilms of the biofilm-forming bacterial species were compared to analyze whether BF depended on the bacterial species. Furthermore, the mean BF with ES (per concentration) and the mean of the 8-day-old biofilm without ES were compared. For the third research question, one-way ANOVA was used and followed by the least significance difference post hoc test. All analyses met the assumption of homogeneity of variances (Levene's statistic: $p>0.05$ ). To analyze whether the BF depended on the material surface, the mean quantity of a 7-day-old biofilm was compared among the materials for each bacterium. The mean quantities of biofilms formed on Days 5, 7, and 9 for each bacterium were analyzed using repeated-measures ANOVA followed by the Bonferroni correction to investigate whether the BF changed among these 3 days. All analyses met the assumption of sphericity (Mauchly's test: $p>0.05$ ). All statistical analyses were performed using $\operatorname{SPSS}(\mathbb{R}$ for

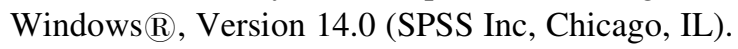

\section{Results}

All tested bacterial species were able to form visible biofilms on PE, SSS, and TI, however biofilms of K. oxytoca, E. faecalis, and E. cloacae could barely be observed, in contrast to the clearly visible biofilms of $\mathrm{S}$. aureus and $\mathrm{S}$. epidermidis. Quantities of biofilm formed by K. oxytoca, E. faecalis, and E. cloacae were insufficient to be measured. Therefore, we focused on BF by $\mathrm{S}$. aureus and $\mathrm{S}$. epidermidis and continued the experiments with these two bacterial species.

BF by $\mathrm{S}$. aureus was determined and compared with BF by $S$. epidermidis. No differences in quantity were seen on PE $(p=0.582)$, SSS $(p=0.051)$, or TI $(p=0.178)$ (Fig. 2).

$\mathrm{BF}$ by $\mathrm{S}$. aureus was greater on $\mathrm{PE}\left(\mathrm{OD}_{595} 0.315\right)$ than on SSS $\left(\mathrm{OD}_{595} 0.190\right)(\mathrm{p}=0.024)$ and $\mathrm{TI}\left(\mathrm{OD}_{595} 0.0386\right)$ $(\mathrm{p}<0.001)$ (Fig. 2A; Table 1) and the same trend was seen for $\mathrm{BF}$ by $\mathrm{S}$. epidermidis $\left(\mathrm{PE}\right.$ : $\mathrm{OD}_{595} 0.248$, SSS: $\mathrm{OD}_{595} 0.104$ [p $\left.<0.001\right]$; TI: $\mathrm{OD}_{595} 0.0578$ [p $\left.\left.<0.001\right]\right)$ (Fig. 2B; Table 2).

S. aureus and $\mathrm{S}$. epidermidis were observed for $\mathrm{BF}$ during 3, 5, 7, and 9 days. On Day 3, biofilms of both bacteria occurred in insufficient quantity to be measured. On TI, PE, and SSS, most biofilm was formed by S. aureus 

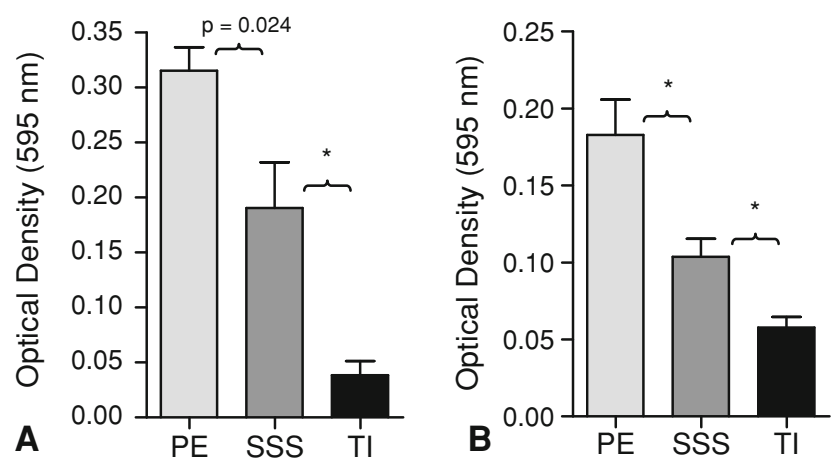

Fig. 2A-B The ODs reflect the quantities of 7-day-old biofilms produced by (A) S. aureus and (B) S. epidermidis on PE, SSS, and TI and answer the second and the third research questions, respectively, whether the quantity of $\mathrm{BF}$ differed among $\mathrm{S}$. aureus and $\mathrm{S}$. epidermidis and whether $\mathrm{BF}$ depended on the biomaterial surface. Both bacteria formed equal quantities of BF and showed PE had the highest BF followed by SSS and TI with the lowest quantity of biofilm. *For these comparisons all $\mathrm{p}$ values $<0.001$.

Table 1. Optical density $(595 \mathrm{~nm})$ for a 7 -day-old biofilm by S. aureus

\begin{tabular}{lll}
\hline Material & 7-day-old biofilm & Background absorbance \\
\hline Polyethylene & 0.315 & 0.167 \\
Surgical stainless steel & 0.190 & 0.101 \\
Titanium & 0.0386 & 0.0801 \\
\hline
\end{tabular}

Table 2. Optical density $(595 \mathrm{~nm})$ for a 7-day-old biofilm by S. epidermidis

\begin{tabular}{lll}
\hline Material & 7-day-old biofilm & Background absorbance \\
\hline Polyethylene & 0.248 & 0.162 \\
Surgical stainless steel & 0.104 & 0.0800 \\
Titanium & 0.0578 & 0.0740 \\
\hline
\end{tabular}

on Day 7 and Day 9 (Day 7 versus Day 9 [all p values $\geq 0.198]$ ) (Fig. 3A). The amount of biofilm formed by $\mathrm{S}$. epidermidis on PE was equal at all times of incubation (Day 5 versus Day 7 [p $=1.000]$, Day 5 versus Day 9 [p = 0.074]) (Fig. 3B). On Day 7 and Day 9 the greatest quantity of biofilms was formed by S. epidermidis on SSS and TI (Day 7 versus Day 9 [all $p$ values $\geq 0.141$ ]) (Fig. 3B).

The addition of ES to 7-day-old biofilms of S. aureus and S. epidermidis reduced these biofilms compared with biofilms without addition of ES on all tested biomaterials (Figs. 4, 5). The biofilm of S. aureus on SSS and PE decreased for all concentrations of ES to minimums of $40.5 \%$ on SSS for $0.31 \mu \mathrm{g}$ ES per well $(\mathrm{p}<0.001)$ and $39.2 \%$ on PE for $0.93 \mu \mathrm{g}$ ES per well $(\mathrm{p}<0.001)$ as compared with the control biofilm without ES (Fig. 4A-B).
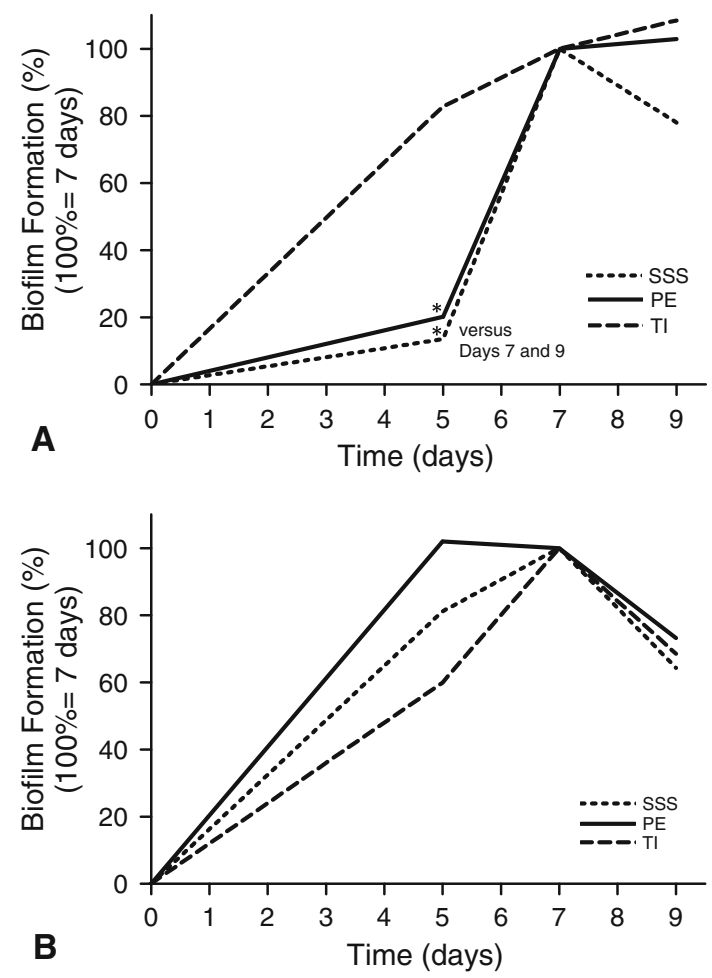

Fig. 3A-B The graphs show the amount of BF after various incubation times and answer the fourth research question, whether quantity of biofilms differed among 3,5, 7, or 9 days. The 7-day-old $\mathrm{BF}$ was defined as $100 \%$. (A) Biofilms by S. aureus kept on growing until Day 9 on PE, TI, and SSS. (B) The largest amount of biofilm by S. epidermidis was formed within 7 to 9 days on SSS and TI. The amount of biofilm on PE was similar at all incubation times. *For these comparisons all $\mathrm{p}$ values $<0.001$; all other comparisons were similar.

On TI, only $8.33 \mu \mathrm{g}$ ES per well showed a reduction $(p=0.005)$ of biofilm (Fig. 4 C), but BF on TI was very low overall. Biofilms of S. epidermidis formed on SSS and $\mathrm{PE}$ also were reduced for all concentrations of ES (Fig. 5A-B). Biofilms decreased to minimums of $32.3 \%$ on SSS for $25 \mu \mathrm{g}$ ES per well $(\mathrm{p}<0.001)$ and $7.6 \%$ on PE for $8.33 \mu \mathrm{g}$ ES per well $(\mathrm{p}<0.001)$ compared with the control. On TI, concentrations of $0.31 \mu \mathrm{g}$ ES per well $(\mathrm{p}<0.001)$ and $0.93 \mu \mathrm{g}$ ES per well $(\mathrm{p}=0.021)$ showed less biofilm (Fig. 5C).

\section{Discussion}

Biofilm-associated infections related to implants cannot be treated easily and frequently the implanted device must be removed with severe consequences for the patient. Therefore, new approaches for these kinds of infections are needed. Cazander et al. previously reported maggot ES reduced $\mathrm{BF}$ of $\mathrm{P}$. aeruginosa [6]. In the current study, we investigated whether other bacterial species, namely, 


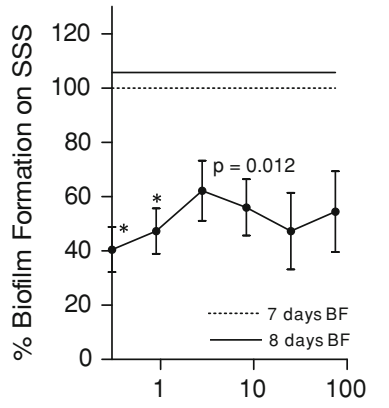

A Concentration ES ( $\mu \mathrm{g} /$ well)
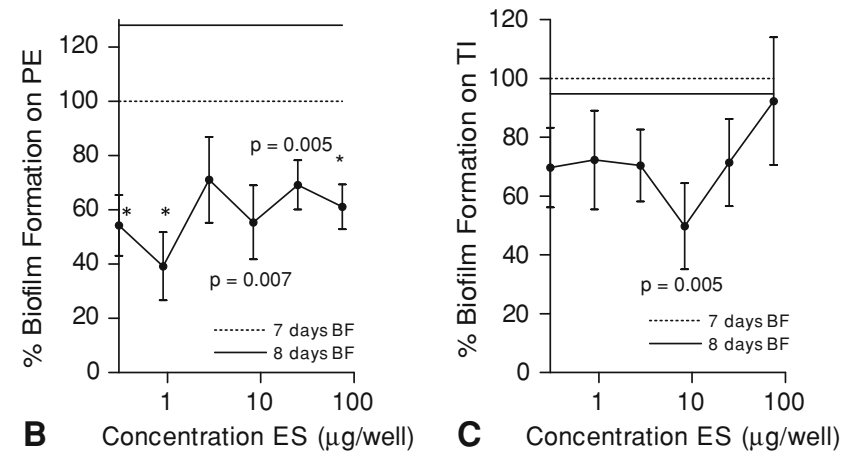

Fig. 4A-C These graphs compare the mean BF produced by $\mathrm{S}$. aureus with ES and the mean control BF after 8 days without ES and answer the last research question whether ES reduced biofilms produced by S. aureus. The 7-day-old biofilm was defined as $100 \%$. All values lower than the 8-day-old biofilm showed biofilm reduction. (A) The biofilm on SSS was decreased for all concentrations with a maximum of $59.5 \%$ for $0.31 \mu \mathrm{g}$ ES per well. (B) The biofilm on PE also was reduced for all concentrations with a maximum of $60.8 \%$ for $0.93 \mu \mathrm{g}$ ES per well. (C) On TI, only $8.33 \mu \mathrm{g}$ ES per well showed biofilm reduction. *For these comparisons all $\mathrm{p}$ values $<0.001$; all other comparisons were similar.

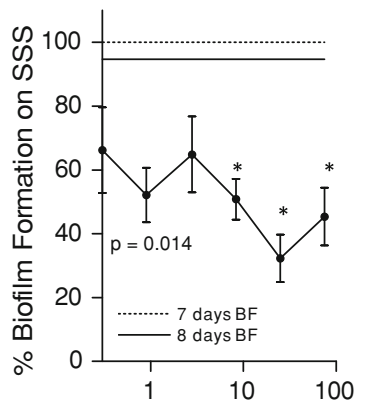

A Concentration ES ( $\mu \mathrm{g} /$ well)

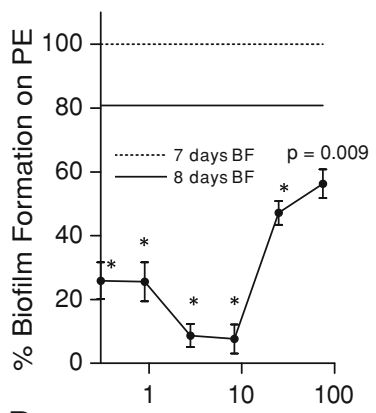

B Concentration ES $(\mu \mathrm{g} /$ well $)$

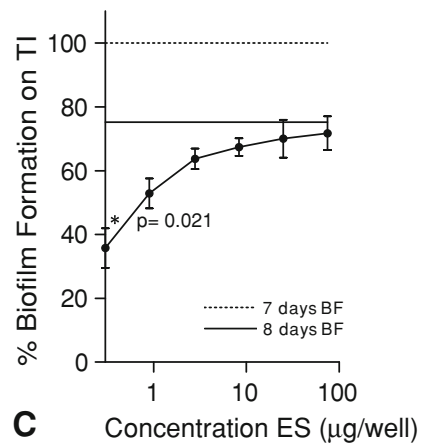

a maximum of $67.7 \%$ for $25 \mu \mathrm{g}$ ES per well. (B) Biofilm on PE also was decreased for all concentrations with a maximum of $92.4 \%$ for $8.33 \mu \mathrm{g}$ ES per well. (C) On TI, only $0.31 \mu \mathrm{g}$ ES per well and $0.93 \mu \mathrm{g}$ ES per well showed biofilm reduction. *For these comparisons all $\mathrm{p}$ values $<0.001$; all other comparisons were similar.

not likely influence the conclusions of this research, because in an earlier study, the effectiveness of ES was compared and similar results of biofilm reduction occurred using various pools of ES [6]. Our study conditions were adapted as much as possible to those in clinical practice. For example, ES were collected under standard conditions in darkness, had an acidity of $\mathrm{pH} 8$, and were incubated at $35^{\circ} \mathrm{C}$. Furthermore, the protein concentration from each collected ES was measured to standardize the various pools. The biomaterials were produced according to specifications for common implants for patients and all bacterial species were isolated from implant infections of patients in our clinic. Therefore, we believe these results were representative and sterile maggot ES reduce not only biofilms of P. aeruginosa [6] but also existing and mature biofilms of $\mathrm{S}$. aureus and $\mathrm{S}$. epidermidis.

We observed biofilms were formed by all tested bacterial species. These bacterial species were expected to form 
biofilms, as they all were isolated from device-related infections. The possible biofilm-forming capacities of the five tested species on medical devices have been described by others $[4,10,20]$. BF by K. oxytoca, E. faecalis, and E. cloacae was insufficient to be quantified. Nutrient media other than BHI were used to test BF by these bacteria but did not show BF. The highest amount of biofilm was formed by S. aureus and S. epidermidis, which are the most frequently isolated pathogens from orthopaedic surgical wounds [1].

$\mathrm{BF}$ between $\mathrm{S}$. aureus and $\mathrm{S}$. epidermidis were similar. One study has compared the quantity among these two bacterial species. Hudetz et al. [12] described equal BF but a stronger adherence to material surfaces by $\mathrm{S}$. epidermidis.

The quantity of BF depended on the biomaterial surface, however published studies show differing results. Hudetz et al. [12] found that the kind of metal, TI or SSS, plays a minor role in the quantity of BF, whereas MacKintosh et al. [16] reported biomaterial surface characteristics do influence BF. We found TI had the least amount of biofilm in vitro formed by S. aureus and S. epidermidis. In a previous study, less biofilm was formed by $\mathrm{P}$. aeruginosa on SSS than on TI [6]. Therefore, we conclude that the quantity of $\mathrm{BF}$ depends on the biomaterial and on the bacterial specie creating the biofilm.

After 7 days of BF, the highest amount of biofilm was formed. In the first 7 days, the nutrient medium in the microtiter plate was not changed, because the biofilm bacteria were still viable as the biofilm kept growing. New nutrient medium with or without ES was added after 7 days of incubation to maintain the viability of the bacteria. The average time for biofilm maturation is 6 to 8 days $[10,22]$. Although the 7-day-old biofilms in this study were not examined by confocal microscopy, their maturation process was more advanced than that of the previously tested 24-hour-old PAO1-biofilms which were decreased by ES [6]. Thus, this research shows more evidence for the therapeutic potential of ES for treatment against biofilmassociated infections which involve mature biofilms.

The biofilm-decreasing capacity of maggot ES seems optimal in low protein concentrations from 0.31 up to $8.33 \mu \mathrm{g}$ ES per well (3.1 to $83.3 \mu \mathrm{g}$ ES per $\mathrm{mL}$ ). In previous studies, protein concentrations of as much as $20 \mu \mathrm{g}$ ES per well were reported as the most effective concentrations for biofilm reduction $[6,28]$. There are several possible explanations for the fact that the influence of ES at higher concentrations than $20 \mu \mathrm{g}$ ES per well declined [6]. We hypothesize a cofactor necessary to regulate the process was depleted [17] or there is a nonenzymatic interaction. In future studies concentration ranges less than $0.31 \mu \mathrm{g}$ ES per well will be tested to examine the lower limit amount of ES causing biofilm reduction.
Previous research suggests maggot ES do not possess direct bactericidal or bacteriostatic activity against planktonic organisms [5]. Therefore, ES are not expected to reduce biofilm by destroying the bacteria in the matrix. However, the influence of ES on biofilm bacteria will be investigated in future studies, by examination of total viable counts of bacteria.

Maggot ES broke down existing biofilms, because all measurements were lower than the control BF after 7 days. However, they also seem to inhibit additional growth of $\mathrm{BF}$ as ES reduced BF to less than $100 \%$ in the experiments with a greater amount of biofilm on Day 8 compared with Day 7 (Fig. 4A-B). Therefore, potential therapeutic use of ES could be prevention or inhibition of $\mathrm{BF}$, eg, flushing surgical wounds before closing and treatment against existing biofilm-associated infections, especially on orthopaedic medical devices. Further research is necessary to clarify the mechanism(s) of biofilm reduction by maggot ES and identify the substance(s) in ES responsible for these reductions.

Acknowledgments We thank Corry Dorresteijn, Janity Pawiroredjo, and Lennaert Renwarin for assistance in the laboratory and Ron Otsen for the photographs.

Open Access This article is distributed under the terms of the Creative Commons Attribution Noncommercial License which permits any noncommercial use, distribution, and reproduction in any medium, provided the original author(s) and source are credited.

\section{References}

1. Arciola CR, Cervellati M, Pirini V, Gamberini S, Montanaro L Staphylococci in orthopaedic surgical wounds. New Microbiol. 2001;24:365-369.

2. Baer WS. The treatment of chronic osteomyelitis with the maggot (larvae of the blowfly). J Bone Joint Surg. 1931;13:438-475.

3. Borriello G, Werner E, Roe F, Kim AM, Ehrlich GD, Stewart PS. Oxygen limitation contributes to antibiotic tolerance of Pseudomonas aeruginosa in biofilms. Antimicrob Agents Chemother. 2004;48:2659-2664.

4. Broomfield RJ, Morgan SD, Khan A, Stickler DJ. Crystalline bacterial biofilm formation on urinary catheters by urease-producing urinary tract pathogens: a simple method of control. J Med Microbiol. 2009;58:1367-1375.

5. Cazander G, van Veen KE, Bernards AT, Jukema GN. Do maggots have an influence on bacterial growth? A study on the susceptibility of strains of five different bacterial species to maggots of Lucilia sericata and their excretions/secretions. J Tissue Viability. 2009;18:80-87.

6. Cazander G, van Veen KE, Bouwman LH, Bernards AT, Jukema $\mathrm{GN}$. The influence of maggot excretions on PAO1 biofilm formation on different biomaterials. Clin Orthop Relat Res. 2009;467:536-545.

7. Costerton JW. Biofilm theory can guide the treatment of devicerelated orthopaedic infections. Clin Orthop Relat Res. 2005; 437:7-11. 
8. Costerton JW. Introduction to biofilm. Int J Antimicrob Agents. 1999;11:217-221.

9. Costerton JW, Stewart PS, Greenberg EP. Bacterial biofilms: a common cause of persistent infections. Science. 1999;284:13181322.

10. Dice B, Stoodley P, Buchinsky F, Metha N, Ehrlich GD, Hu FZ. Biofilm formation by ica-positive and ica-negative strains of Staphylococcus epidermidis in vitro. Biofouling. 2009;25:367375.

11. Giacometti A, Cirioni O, Schimmizi AM, Del Prete MS, Barchiesi F, D'Errico MM, Petrelli E, Scalise G. Epidemiology and microbiology of surgical wound infections. J Clin Microbiol. 2000;38:918-922.

12. Hudetz D, Ursic Hudetz S, Harris LG, Luginbühl R, Friederich NF, Landmann R. Weak effect of metal type and ica genes on staphylococcal infection of titanium and stainless steel implants. Clin Microbiol Infect. 2008;14:1135-1145.

13. Jukema GN, Menon AG, Bernards AT, Steenvoorde P, Taheri Rastegar A, Van Dissel JT. Amputation-sparing treatment by nature: "surgical" maggots revisited. Clin Infect Dis. 2002;35: $1566-1571$.

14. Kadurugamuwa JL, Francis KP. Bioluminescent imaging of bacterial biofilm infections in vivo. Methods Mol Biol. 2008;431: 225-239.

15. Levy SB. The challenge of antibiotic resistance. Sci Am. 1998;278:46-53.

16. MacKintosh EE, Patel JD, Marchant RE, Anderson JM. Effects on biomaterial surface chemistry on the adhesion and biofilm formation of Staphylococcus epidermidis in vitro. J Biomed Mater Res A. 2006;78:836-842.

17. Meisenberg G, Simmons WH. Principles of Medical Biochemistry. St Louis, MO: Mosby;1998.

18. Nablo BJ, Prichard HL, Butler RD, Klitzman B, Schoenfisch MH. Inhibition of implant-associated infections via nitric oxide release. Biomaterials. 2005;26:6984-6990.

19. Pitts B, Hamilton MA, Zelver N, Stewart PS. A microtiter-plate screening method for biofilm disinfection and removal. J Microbiol Methods. 2003;54:269-276.
20. Sandoe JA, Witherden IR, Cover JH, Heritage J, Wilcox MH. Correlation between enterococcal biofilm formation in vitro and medical-device-related infection potential in vivo. $J$ Med Microbiol. 2003;52:547-550.

21. Sherman RA, Pechter EA. Maggot therapy: a review of the therapeutic applications of fly larvae in human medicine, especially for treating osteomyelitis. Med Vet Entomol. 1988;2:225230.

22. Sousa C, Henriques M, Teixeira P, Oliveira R. Relevance of cell wall and extracellular matrix proteins to Staphylococcus epidermidis adhesion and biofilm formation. J Adhes Sci Techn. 2009;23:1657-1671.

23. Stepanovic S, Vokovic D, Dakic I, Savic B, Svabic-Vlahovic M. A modified microtiter-plate test for quantification of staphylococcal biofilm formation. J Microbiol Methods. 2000;40:175179.

24. Stewart PS, Costerton JW. Antibiotic resistance of bacteria in biofilms. Lancet. 2001;358:135-138.

25. Tantawi TI, Gohar YM, Kotb MM, Beshara FM, El-Naggar MM. Clinical and microbiological efficacy of MDT in the treatment of diabetic foot ulcers. J Wound Care. 2007;16:379-383.

26. Trampuz A, Widmer AF. Infections associated with orthopedic implants. Curr Opin Infect Dis. 2006;19:349-356.

27. US Food and Drug Administration. General Plastic Surgery Devices Panel July 27, 2005. Briefing Information. Panel Classification Memo. Available at: www.fda.gov/ohrms/dockets/ac/ 05/briefing/2005-4168b1.htm Accessed August 25, 2005.

28. van der Plas MJ, Jukema GN, Wai SW, Dogterom-Ballering HC, Lagendijk EL, van Gulpen C, van Dissel JT, Bloemberg GV, Nibbering PH. Maggot excretions/secretions are differentially effective against biofilms of Staphylococcus aureus and Pseudomonas aeruginosa. J Antimicrob Chemother. 2008;61:117-122.

29. Wainwright M. Miracle Cure: The Story of Penicillin and the Golden Age of Antibiotics. Oxford, UK: Basil Blackwell; 1990.

30. Woodworth BA, Tamashiro E, Bhargave G, Cohen NA, Palmer JN. An in vitro model of Pseudomonas aeruginosa biofilms on viable airway epithelial cell monolayers. Am J Rhinol. 2008;22:235-238. 